\title{
Giriş Kısıtlı Ters Sarkaç Mekanizmasının Optimal Kontrolü
}

\author{
Sait Sovukluk ${ }^{1,2, *}$, Mustafa Mert Ankaral1 ${ }^{3, \neq}$ \\ ${ }^{1}$ Middle East Technical University, Faculty of Engineering, Department of Electrical and Electronics Engineering, Ankara, Turkey, (ORCID: 0000-0002-8698-8835), \\ sovukluk.sait@metu.edu.tr \\ 2 Teknolus Enerji Ltd. Şti., Robotik ve Yazılım Birimi, Ankara, Turkey \\ ${ }^{3}$ Middle East Technical University, Faculty of Engineering, Departmant of Electrical and Electronics Engineering, Ankara, Turkey, (ORCID: 0000-0002-1235-5373), \\ mertan@metu.edu.tr
}

(Ilk Geliş Tarihi 17 Mart 2021 ve Kabul Tarihi 27 Haziran 2021)

(DOI: 10.31590/ejosat.898753)

ATIF/REFERENCE: Sovukluk, S., Ankaral1, M.M. (2021). Optimal Control of Input Constrained Inverted Pendulum. Avrupa Bilim ve Teknoloji Dergisi, (25), 247-255.

$\ddot{O} \mathbf{z}$

Günümüz eyleyici ve algılayıcı teknolojilerindeki hızı gelişmeler, çok farklı eyleyici ve algılayıcı kombinasyonları içeren platformlarının geliştirilmesine öncülük etmiştir. Bahsi geçen platformların potansiyellerini en iyi şekilde değerlendirebilmek amacıyla kontrol sistemlerinde yeni metotların geliştirilmesi bir gereklilik haline gelmiş ve yeni kontrol metotları modern kontrol adı altında toplanmaya başlamıştır. Modern kontrolün alt başlıklarından biri olan optimal kontrol, bilgisayarların işlem güçlerinin artmasıyla birlikte günümüzde çok yaygın olarak kullanılmaya başlanmıştır. Bu çalışmada klasik kontrol metotlarına kıyasla çeşitli avantajları olan optimal kontrolün alt başlıklarından dört tanesi olan sonlu ufuklu LQR, sonsuz ufuklu LQR, sonlu ufuklu MPC ve ikili mod MPC kontrolcülerinin uygulamaları incelenmiştir. Bu inceleme dijital bilgisayarların doğasına uygun olarak süreksiz zaman uzayında gerçekleştirilmiştir. Bahsi geçen dört kontrolcünün kontrol performanslarının gözlenebilmesi amacıyla bir ters sarkaç sistem modeli kullanılmıştır. Ters sarkaç mekanizması doğrusal olmayan, stabil olmayan ve eklem sayısından daha az sayıda eyleyiciye sahip olan yapısı sebebiyle kapsayıcı bir dinamik modeldir. Bu çalışmayı incelemesinin ardından okuyucu bu dört kontrol sistemleri arasından hangisinin hangi durumlarda nasıl uygulanması gerektiği hakkında fikir sahibi olacaktır.

Anahtar Kelimeler: Süreksiz zamanlı sonlu ufuklu LQR kontrolü, süreksiz zamanlı sonsuz ufuklu LQR kontrolü, süreksiz zamanlı sonlu ufuklu MPC kontrolü, süreksiz zamanlı ikili mod MPC kontrolü, ters sarkaç mekanizması.

\section{Optimal Control of Input Constrained Inverted Pendulum}

\begin{abstract}
Rapid developments in today's actuator and sensor technologies have pioneered the development of platforms containing very different actuator and sensor combinations. In order to utilize the potentials of the platforms in the best way, it has become a necessity to develop new methods in control systems and new control methods have begun to be gathered under the name of modern control. The optimal control, which is one of the subtitles of modern control, has started to be used widely today with the increase in the processing power of computers. In this study, applications of finite horizon LQR, infinite horizon LQR, finite horizon MPC and dual mode prediction MPC controllers, which are four of the subtitles of optimal control, which have various advantages compared to classical control methods, have been investigated. This investigation was carried out in discrete time space in accordance with the nature of digital computers. An inverted pendulum system model is used to observe the control performances of the four controllers. The inverted pendulum mechanism is an inclusive dynamic model due to its nonlinear, unstable and underactuated nature. After reviewing this study, the reader will have an idea of which of these four control systems should be applied in which situations.
\end{abstract}

Keywords: Discrete time finite horizon LQR controller, discrete time infinite horizon LQR controller, discrete time finite horizon MPC controller, discrete time dual-mode prediction MPC controller, inverted pendulum.

*Sorumlu Yazar: sovukluk.sait@metu.edu.tr 


\section{Giriş}

Klasik kontrol sistem tasarımlarının önemli bir bölümü kabul edilebilir bir sistem performansı elde edilene kadar farklı analiz metotları kullanılarak yapılan tekrarlamalı bir denemeyanılma sürecidir. Bu tarz kapalı döngü kontrol sistemlerinde sistem performansı genellikle yükselme zamanı, aşma miktarı, bant genişliği vs. gibi kriterlerle ölçülür. Ek olarak, genellikle tek referans girdili ve tek durum çıkışlı sistemlerde kullanılan klasik kontrol metotları modern teknolojinin gerektirdiği kontrol ihtiyaçlarını karşılamakta yetersiz kalabilmektedir. Örneğin, bir uzay mekiğinin irtifa kontrolünün, harcanan yakıt miktarının minimum seviyede tutularak yapılması doğrudan klasik kontrol metotlarıyla sağlanabilecek bir yöntem değildir. Dijital bilgisayarların yaygınlaşması ve işlem güçlerinin artmasıyla birlikte modern teknoloji ihtiyaçlarını karşılayan, çok referans girdili ve çok durum çıktılı sistemlerin kontrolü için uyumlu, uzay mekiğindeki örnek gibi kaynak kullanımını minimumda tutabilecek bir kontrol yöntemi olan optimal kontrol çalışılması önem arz eden bir konu olmuştur (Kirk, 2004).

Optimal kontrol sistemleri günümüz teknolojisi olan insansız hava araçlarının kontrolünde (Arifianto ve Farhood, 2015; Kamel ve ark., 2017), sürü robotiği çalışmalarında (Zhang ve ark., 2015), karmaşık bir dinamik yapıya sahip olduğu bilinen dört bacaklı robotların yürüme ve akrobasi hareketlerinin kontrolünde (Ding ve ark., 2019; Villarreal ve ark. 2020), otonom araçların yörünge planlaması ve kontrolünde (Batkovic ve ark., 2019), kara araçları için geliştirilen engellerden kaçınma uygulamalarında (Frasch ve ark., 2013), helikopter iniş kontrolünde (Greer ve Sultan, 2020), yakıt tasarruflu roket iniş kontrolünde (Wang ve ark., 2019) kullanım örnekleri bulunan, popüler bir kontrol metodudur. Verilen çalışma örneklerinde de görüldüğü üzere özellikle MPC kontrolcüsünün, sistem limitlerini göz önünde bulundurması sebebiyle, çok farklı alanlarda kullanılabildiği görülmektedir. Ek olarak, en bilindik optimal kontrol metotlarından biri olan LQR kontrolcüsünün, yine en bilindlik klasik kontrol metotlarından biri olan PID kontrolcüsü yerine tercih edilmesini öneren çeşitli çalışmalar da bulunmaktadır (Akgul ve ark., 2012; Çeven ve Albayrak, 2020).

Optimal kontrolün popülerliği ve kanıtlanan avantajları bu konuyu çalışmaya değer kılmaktadır. Yaptığımız çalışmada optimal kontrolün alt başlıklarından dördü olan sonlu ufuklu LQR, sonsuz ufuklu LQR, sonlu ufuklu MPC ve ikili mod MPC kontrolcülerinin hangi durumlarda kullanılmasının daha avantajlı olduğu irdelenmiştir. Bu çalışma sonucunda, optimal kontrolü bir platforma uygulamak isteyen okuyucu, fiziksel sisteminin yapısına, kullanılabilir işlem gücüne ve kontrol ihtiyaçlarına göre yukarıda belirtilen dört kontrolcü arasından hangisini, nasıl uygulaması gerektiği hakkında fikir sahibi olacaktır.

Bahsi geçen dört kontrolcünün kontrol performanslarının gözlenebilmesi amacıyla bir ters sarkaç sistem modeli kullanılmıştır. Ters sarkaç mekanizması doğrusal olmayan, stabil olmayan ve eklem sayısından daha az sayıda eyleyiciye sahip olan yapısı sebebiyle kapsayıcı bir dinamik modeldir. Bu kapsayıcılığına ek olarak, çoğu okuyucunun aşina olduğu düşünülen, aşina olunmasa bile sezgisel/matematiksel olarak anlaşılması kolay olan bu dinamik model üzerinden kontrol sisteminin ve mekanizmanın tepkilerine anlam vermek daha kolay olacaktır.

\section{Sistem Modeli}

Kontrol edilecek ters sarkaç mekanizmasının serbest cisim diyagramı Şekil 1' de gösterilmiştir. Ters sarkaç sisteminin arabası dışarıdan uygulanan bir kuvvet ile tek eksende ileri geri hareket ettirilmekte ve arabaya bağlı olan sarkaç ise bahsedilen hareket sonucunda sarkaç açısı olan $\theta$ açısı sıfır olacak şekilde tutulmaya çalışılmaktadır. Sisteme dışarıdan uygulanan tek kuvvet arabaya $\mathrm{x}$-doğrultusunda uygulanan kuvvettir. Sarkaç, arabaya serbest bir eklem ile bağlanmakta ve bu eklemde eyleyici bulunmamaktadır. Mekanizmadaki arabanın kütlesi 1 $\mathrm{kg}$, sarkacın kütlesi $0,5 \mathrm{~kg}$ ve sarkacın uzunluğu ise $0,3 \mathrm{~m}$ olarak modellenmiştir.

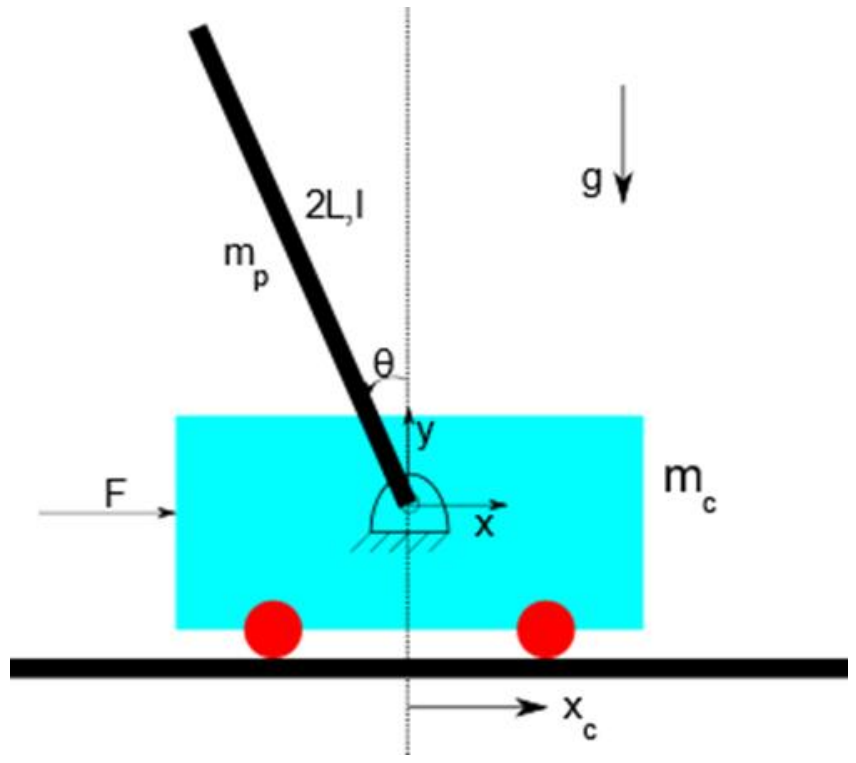

Şekil 1. Ters sarkaç sisteminin serbest cisim diyagramı.

Ters sarkaç sisteminin hareket denklemlerinin türetilmesi sonucunda elde edilen ve Eş. 1' de gösterilen formatta bulunan durum uzayı Eş. 2' de gösterilmiştir. Eğer n durum sayısını, p kontrol sinyali sayısını ve q çıktı sayısını ifade ederse, Eş. 1' de gösterilen $\boldsymbol{x}(t) \in \mathbb{R}^{n}$ durum vektörünü, $\boldsymbol{A} \in \mathbb{R}^{n \times n}$ karakteristik matrisini, $\boldsymbol{B} \in \mathbb{R}^{n \times p}$ kontrol matrisini, $\boldsymbol{u}(t) \in \mathbb{R}^{p}$ kontrol vektörünü, $\boldsymbol{y}(t) \in \mathbb{R}^{q}$ çıktı vektörünü, $\boldsymbol{C} \in \mathbb{R}^{q \times n}$ çıktı matrisini gösterir. Modelleme sirasında sistemde enerji kaybı olmadığı, hareketin tamamen düzlemsel olduğu, teker ile zemin arasında kayma olmadığı ve teker ile zeminin her zaman temas halinde olduğu varsayımları yapılmıştır. Son olarak da elde edilen doğrusal olmayan denklemler, yapılan varsayımlar doğrultusunda ve sistemin sıfır $\theta$ açısı etrafinda küçük açı hareketleri yapacağı varsayımı ile doğrusal hale getirilmiştir. Sarkaç açısının sıfir dereceden uzaklaşması kontrolcü tasarımı için kullanılan modeli doğruluktan saptırmaktadır. Durum uzayı gösteriminde dikkat çeken bir detay sistemin iki çıktısının olmasıdır. Yani sarkacın açısına ek olarak arabanın pozisyonu da sistemin çıktıları arasında yer almaktadır. Arabanın hareket edecek sonsuz alanı olmadığından dolayı arabanın pozisyonunun da kontrol edilmesi gerekmektedir. Durum uzayı gösteriminde dikkat çeken bir diğer nokta ise sisteminin tamamen yönetilebilir ve gözlenebilir olmasıdır. Sistemin tamamen yönetilebilir olması herhangi bir durum değişkeninin kontrol sinyali ile herhangi bir değerden başka bir değere ulaştırılabileceğini ifade eder. Buna benzer olarak sistemin tamamen gözlenebilir olması da sistemin çıktıları olan sarkaç açısı ve araba pozisyonu bilgileri ile diğer herhangi bir durum değişkeninin hesaplanabileceğini ifade eder. 
Eş. 2' de gösterilen sürekli zamanlı durum uzayını, süreksiz zamanlı durum uzayına dönüştürmek için Eş. 3 kullanılabilir. Eşlenikte gösterilen $\boldsymbol{A}_{\boldsymbol{d}}, \boldsymbol{B}_{\boldsymbol{d}}, \boldsymbol{C}_{\boldsymbol{d}}$ matrisleri, sürekli zamanlı durum uzayındaki eşleniklerinin süreksiz zamana dönüştürülmüş halini, $\mathrm{T}$ ise süreksizliğin periyodunu simgelemektedir. Süreksiz zaman uzayında çalışan bilgisayar ortamlarında durum uzaylı benzetimlerin ve tasarımların süreksiz zamanlı durum uzayı modeli ile yapılması daha doğru sonuçlar elde edilmesini sağlayacaktır. $\mathrm{Bu}$ çalışmada kontrolcü tasarımında kullanılan durum uzayı süreksiz zamanlıdır.

$$
\begin{aligned}
& \dot{x}=A x+B u \\
& y=C x \\
& {\left[\begin{array}{l}
\dot{x}_{1}(t) \\
\dot{x}_{2}(t) \\
\dot{x}_{3}(t) \\
\dot{x}_{4}(t)
\end{array}\right]=\left[\begin{array}{c}
\dot{x}_{c}(t) \\
\ddot{x}_{c}(t) \\
\dot{\theta}(t) \\
\ddot{\theta}(t)
\end{array}\right]=\left[\begin{array}{cccc}
0 & 1 & 0 & 0 \\
0 & 0 & \frac{m_{p}^{2} g L^{2}}{I\left(m_{p}+m_{c}\right)+m_{p} m_{c} L^{2}} & 0 \\
0 & 0 & 0 & 1 \\
0 & 0 & \frac{m_{p} g L\left(m_{p}+m_{c}\right)}{I\left(m_{p}+m_{c}\right)+m_{p} m_{c} L^{2}} & 0
\end{array}\right]\left[\begin{array}{c}
x_{1}(t) \\
x_{2}(t) \\
x_{3}(t) \\
x_{4}(t)
\end{array}\right]} \\
& +\left[\begin{array}{c}
0 \\
\frac{I+m_{p} L^{2}}{I\left(m_{p}+m_{c}\right)+m_{p} m_{c} L^{2}} \\
0 \\
\frac{m_{p} L}{I\left(m_{p}+m_{c}\right)+m_{p} m_{c} L^{2}}
\end{array}\right] u(t) \\
& y(t)=\left[\begin{array}{llll}
1 & 0 & 0 & 0 \\
0 & 0 & 1 & 0
\end{array}\right]\left[\begin{array}{c}
\dot{x}_{c}(t) \\
\ddot{x}_{c}(t) \\
\dot{\theta}(t) \\
\ddot{\theta}(t)
\end{array}\right]+\left[\begin{array}{l}
0 \\
0
\end{array}\right] u(t) \\
& \boldsymbol{A}_{\boldsymbol{d}}=e^{\boldsymbol{A} T}, \quad \boldsymbol{B}_{\boldsymbol{d}}=\int_{0}^{T} e^{\boldsymbol{A} \tau} d \tau, \quad \boldsymbol{C}_{\boldsymbol{d}}=\boldsymbol{C}
\end{aligned}
$$

\section{Kontrolcü Modelleri}

Belirtildiği gibi sarkaç açısı olan $\theta$ açısını sıfır derecede tutmak ve arabayı istenen pozisyonda tutabilmek amaciyla sisteme kontrolcü eklenmesi gerekmektedir. $\mathrm{Bu}$ kontrolcü, hedeflenen davranışlar için gerekli olan kuvvet girişini hesaplayacak ve bu kuvveti sisteme uygulayacaktır. Tasarlanan kontrol sisteminin blok diyagram gösterimi Şekil 2' de gösterilmiştir. Sürekli zamanda olan ters sarkaç mekanizması ile süreksiz zamanda olan kontrol sistemi arasındaki sinyal dönüşümleri belirtilen şekil içerisinde ayrıca gösterilmiştir. Farklı optimal kontrolcülerin irdelendiği bu kısımda sırası ile sonlu ufuklu LQR kontrolcüsü, sonsuz ufuklu LQR kontrolcüsü, sonlu ufuklu MPC kontrolcüsü ve son olarak da ikili mod MPC kontrolcüsü incelenecektir. Bahsi geçen dört kontrolcü de Şekil 2' de gösterilen dijital kontrol bloğunu kendilerine has yöntemlerle şekillendirmektedirler.

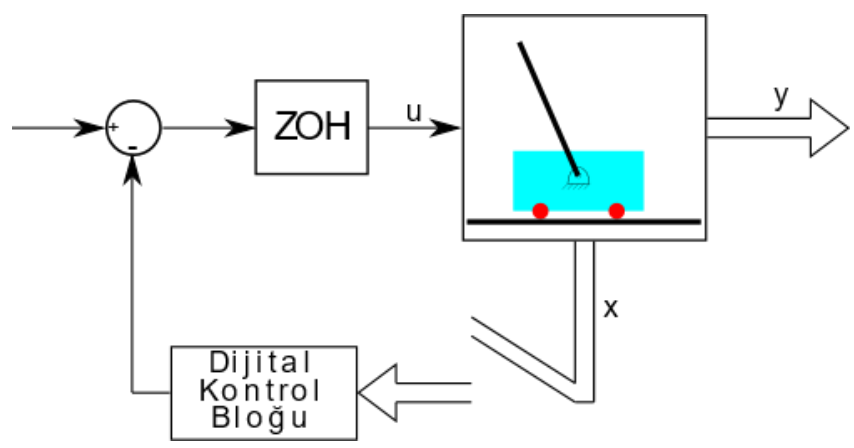

Şekil 2. Kontrol sistemi blok diyagramı yapısı

\subsection{Süreksiz Zamanlı Sonlu Ufuklu LQR Kontrolcüsü}

Sonlu ufuklu LQR kontrolcüsü, sistemin bulunulan zamandaki durumunu ve gelecekteki sonlu sayıdaki durumlarının süreksiz zamanlı durum uzayı modeli ile elde edilen tahminini bir bedel fonksiyonunda toplayip, bu bedel fonksiyonunu minimuma getirecek kontrol sinyalini hesaplar ve bunu sisteme uygular. $\mathrm{Bu}$ sayede bedel fonksiyonundaki ağırlıklara göre en optimal kontrol uygulanmış olur. LQR problemi tanımı Eş. 4' de gösterilmiştir. Eş. 4' de gösterilen bedel fonksiyonunun dikkat çeken bir özelliği kuadratik formda olmasıdır. Bedel fonksiyonunun kuadratik (ikinci dereceden) formda olması her zaman bir minimum veya maksimum değerinin olduğunu garanti eder. Ek olarak doğrusal yapılı olan bu bedel fonksiyonunun çözümü de hızlı ve kolaydır. LQR probleminde kullanılan $x(t) \in \mathbb{R}^{n}$ durum vektörünü, simetrik pozitif yarı-tanımlı $Q_{f} \in \mathbb{R}^{n \times n}$ matrisi final basamaktaki durum değişkenlerinin ağırlık katsayısını, simetrik pozitif yarı-tanımlı $Q \in \mathbb{R}^{n \times n}$ matrisi ara basamaklardaki durum değişkenlerinin toplamının ağırlık katsayısını, simetrik pozitif tanımlı $R \in \mathbb{R}^{p \times p}$ matrisi verilecek kontrol sinyalinin ağırlık katsayısını, $N$ hesaba katılacak basamak sayısını, $u(t) \in \mathbb{R}^{p}$ kontrol vektörünü simgeler. Ağırlık katsayıları kontrolcü parametreleridir ve değişkenlerin bedel baskınlığını belirler. Örneğin kontrol sinyalinin ağırlık katsayısının yüksek seçilmesi, kontrol sinyalinin büyük bir bedele sahip olmasına sebep olur. Kontrol sinyalinin büyük bir bedele sahip olması da optimizasyon sonucunda hesaplanan kontrol sinyalinin değerinin küçük olmasına sebep olur, çünkü bedel fonksiyonunun değerinin minimum olması istenmektedir. Benzer şekilde kontrol sinyalinin ağırlık katsayısının küçük seçilmesi kontrolcünün sisteme, kıyasla, daha agresif kuvvetler uygulamasına sebep olur. Kontrol performansı $Q, Q_{f}, R$ ve N parametlerinin seçimine göre değişim göstermektedir.

Sunulan LQR probleminin analitik çözümü durumlara göre büyük matrislerin terslerinin alınmasını gerektirmektedir. Yoğun bir işlem gücü gerektiren matris tersine alma işlemine alternatif olarak problemin çözümü döngülü olarak yapılabilmektedir. Bahsedilen döngülü çözüm Eş. 5' de gösterilmiştir. Bu döngülü çözümün sonuçlandırılması için Eş. 6' da gösterilen geriye özyinelemeli Riccati denkleminin çözülmesi gerekmektedir. Geriye özyinelemeli Riccati denkleminin çözülmesi sonucunda Eş. 5' de gösterilen gerekli kontrol sinyali değerinin çözülüp sisteme uygulanması sonucunda kontrol yapılmış olacaktır. 


$$
\underbrace{\min }_{u_{0}, \ldots, u_{N-1}}\left\{J=\boldsymbol{x}_{N}^{T} \boldsymbol{Q}_{f} \boldsymbol{x}_{N}+\sum_{\text {Bağll olarak: }}^{N-1}\left(\boldsymbol{x}_{k}^{T} \boldsymbol{Q} \boldsymbol{x}_{k}+\boldsymbol{u}_{k}^{T} \boldsymbol{R} \boldsymbol{u}_{k}\right)\right\}
$$

$$
\begin{gathered}
\boldsymbol{x}_{k+1}=\boldsymbol{A} \boldsymbol{x}_{k}+\boldsymbol{B} \boldsymbol{u}_{k}, \quad k=0,1, \ldots, N-1 ; x_{0} \text { biliniyor } \\
\boldsymbol{Q}=\boldsymbol{Q}^{T} \geq 0, \quad \boldsymbol{Q}_{\boldsymbol{f}}=\boldsymbol{Q}_{\boldsymbol{f}}^{T} \geq 0, \quad \boldsymbol{R}=\boldsymbol{R}^{T}>0 \\
\boldsymbol{u}_{k}=-\underbrace{\left(\boldsymbol{R}+\boldsymbol{B}^{T} \boldsymbol{P}_{k+1} \boldsymbol{B}\right)^{-1} \boldsymbol{B}^{T} \boldsymbol{P}_{k+1} \boldsymbol{A}}_{K} \boldsymbol{x}_{k} \\
\boldsymbol{P}_{k}=\boldsymbol{A}^{T} \boldsymbol{P}_{k+1} \boldsymbol{A}+\boldsymbol{Q}-\boldsymbol{A}^{T} \boldsymbol{P}_{k+1} \boldsymbol{B}\left(\boldsymbol{B}^{T} \boldsymbol{P}_{k+1} \boldsymbol{B}+\boldsymbol{R}\right)^{-1} \boldsymbol{B}^{T} \boldsymbol{P}_{k+1} \boldsymbol{A} \\
\boldsymbol{P}_{N}=\boldsymbol{Q}_{\boldsymbol{f}}
\end{gathered}
$$

\subsection{Süreksiz Zamanlı Sonsuz Ufuklu LQR Kontrolcüsü}

Sonlu ufuklu LQR kontrolcüsünden farklı olarak sonsuz ufuklu LQR kontrolcüsü bedel fonksiyonu içerisinde sistemin bulunduğu durumu ve sonlu miktardaki gelecek durumları değil, sistemin bulunduğu durumdan itibaren sonsuz zaman sonraki durumuna kadar olan tüm davranışlarını hesaba katarak bir kontrol sinyali hesaplamayı temel alır. Sonsuz ufuklu LQR probleminin çözümü sonlu ufuklu LQR problemine benzerlik göstermektedir. Çözümler arasındaki farkların biri Eş. 6' da gösterilen geriye özyinelemeli Riccati denkleminin daha farklı bir hal alması, öteki ise geri besleme kazanç matrisinin $\left(\boldsymbol{K}_{\infty} \in\right.$ $\mathbb{R}^{p \times n}$ ) sabit olmasıdır. Eş. 7' de kontrol sinyalinin yeni yapısı, Eş. 8' de ise süreksiz zamanlı cebirsel Riccati denklemi gösterilmiştir.

$$
\begin{gathered}
\boldsymbol{u}_{k}=-\underbrace{\left(\boldsymbol{R}+\boldsymbol{B}^{T} \boldsymbol{P}_{\infty} \boldsymbol{B}\right)^{-1} \boldsymbol{B}^{T} \boldsymbol{P}_{\infty} \boldsymbol{A}}_{K_{\infty}} \boldsymbol{x}_{k} \\
\boldsymbol{P}_{\infty}=\boldsymbol{A}^{T} \boldsymbol{P}_{\infty} \boldsymbol{A}+\boldsymbol{Q}-\boldsymbol{A}^{T} \boldsymbol{P}_{\infty} \boldsymbol{B}\left(\boldsymbol{B}^{T} \boldsymbol{P}_{\infty} \boldsymbol{B}+\boldsymbol{R}\right)^{-1} \boldsymbol{B}^{T} \boldsymbol{P}_{\infty} \boldsymbol{A}
\end{gathered}
$$

\subsection{Süreksiz Zamanlı Sonlu Ufuklu MPC Kontrolcüsü}

Sonlu ufuklu MPC kontrolcüsü, sonlu ufuklu LQR kontrolcüsüne benzer bir bedel fonksiyonu içerir. Buradaki farklılık optimizasyon sirasinda kontrol sinyalindeki ve durum uzayı değişkenlerindeki limitlerin de hesaba katılmasıdır. Optimizasyon sırasında hesaba katılması gereken bu doğrusal olmayan limitler, bahsi geçen LQR kontrolcülerinde kullanılan döngülü çözümlerin kullanım dışı kalmalarına sebep olmakta ve büyük matrislerin terslerinin hesaplanmasını gerektirmektedir. MPC problemlerinde kullanılan yaygın çözüm yöntemi, LQR probleminin en-kü̧̈ük-kareler problemi (least-squares problem) olarak yazılıp kuadratik programlama ile çözülmesidir. Sonlu ufuklu MPC problemi Eş. 9' da gösterilmiş̧ir. LQR kontrolcülerden farklı olarak bu problem çözülürken eşlenik içerisinde belirtilen tüm limitler hesaba katılmalıdır. Eşlenik içerisinde sırasıyla durum uzayı değişkenlerinin limitlerinin, kontrol sinyali değişkenlerinin limitlerinin, durum uzayı değişkenlerinin doğrusal kombinasyonlarının limitlerinin ve kontrol sinyali değişkeninin doğrusal kombinasyonlarının limitlerinin göz önünde bulundurulduğu gösterilmiştir. Ek olarak LQR probleminin gösteriminden farklı olarak indekslerde bir gösterim farklılığı dikkat çekmektedir. $\mathrm{Bu}$ farklılık LQR kontrolcüsünden farklı olarak her basamakta $\mathrm{N}$ kadar basamak ilerisinin simülasyonunun limitler göz önünde bulundurularak yapilmasından kaynaklanmaktadır. Normal bir LQR kontrolcüsünde $\mathrm{N}$ basamak kullanılarak yapılan optimizasyonda
$\mathrm{N}$ basamak için de gerekli olan geri bildirim kazanç matrisi değerleri tek seferde hesaplanabilmektedir. Fakat MPC içerisinde bulunan doğrusal olmayan limitlerin getirdiği zorlukların aşılması için her basamakta $\mathrm{N}$ kadar ilerideki basamağın hesaplamasının tekrar yapılması gerekmektedir. $\mathrm{Bu}$ farkl1lı sonlu ufuklu MPC kontrolcüsüne ek bir hesaplama maliyeti getirmektedir. Sonlu ufuklu MPC kontrolcüsünün dikkat çeken bir detayı ise herhangi bir limitlemenin olmadığı durumlarda (bedel fonksiyonunun doğrusal olduğu durumlarda) kontrolcünün, aynı ufuk sayısina sahip sonlu ufuklu LQR kontrolcüsü ile aynı kontrol performansına sahip olmasıdır. Sisteme eklenen limitlemeler bu iki kontrolcü arasında farklılaşmalara sebep olmaktadır.

$$
\begin{gathered}
\underbrace{\min }_{u_{0 \mid k} \ldots u_{N-1 \mid k}}\left\{J=\boldsymbol{x}_{|k|}^{T} \boldsymbol{Q}_{\boldsymbol{f}} \boldsymbol{x}_{N \mid k}+\sum_{j=0}^{N-1}\left(\boldsymbol{x}_{j \mid k}^{T} \boldsymbol{Q} \boldsymbol{x}_{j \mid k}+\boldsymbol{u}_{j \mid k}^{T} \boldsymbol{R} \boldsymbol{u}_{j \mid k}\right)\right\} \\
\text { Bağll olarak: } \\
\boldsymbol{x}_{k+1}=\boldsymbol{A} \boldsymbol{x}_{k}+\boldsymbol{B} \boldsymbol{u}_{k}, \quad k=0,1, \ldots, N-1 ; \quad x_{0} \text { biliniyor } \\
\boldsymbol{Q}=\boldsymbol{Q}^{T} \geq 0, \quad \boldsymbol{Q}_{\boldsymbol{f}}=\boldsymbol{Q}_{\boldsymbol{f}}^{T} \geq 0, \quad \boldsymbol{R}=\boldsymbol{R}^{T}>0 \\
\boldsymbol{x}_{\boldsymbol{m i n}} \leq \boldsymbol{x}_{j \mid k} \leq \boldsymbol{x}_{\boldsymbol{m a x}}, j=0,1, \ldots, N \\
\boldsymbol{u}_{\boldsymbol{m i n}} \leq \boldsymbol{u}_{j \mid k} \leq \boldsymbol{u}_{\boldsymbol{m a x}}, j=0,1, \ldots, N-1 \\
\boldsymbol{f}_{\boldsymbol{x}}^{T} \boldsymbol{x}_{j \mid k} \leq \boldsymbol{v}_{\boldsymbol{x}}, j=0,1, \ldots, N \\
\boldsymbol{f}_{u}^{T} \boldsymbol{u}_{j \mid k} \leq \boldsymbol{v}_{\boldsymbol{u}}, j=0,1, \ldots, N-1
\end{gathered}
$$

\subsection{Süreksiz Zamanlı İkili Mod MPC Kontrolcüsü}

İkili mod MPC kontrolcüsü, sonsuz ufuklu LQR kontrolcüsü ile sonlu ufuklu MPC kontrolcüsünün birleştirilmesi sonucu elde edilen bir kontrolcüdür. Sonlu ufuklu MPC kontrolcüsünden farklı olarak $\boldsymbol{Q}_{\boldsymbol{f}}$ matrisi yani final basamaktaki durum değişkenlerinin ağıllık katsayısı süreksiz zamanlı Lyapunov denklemi koşulunu sağlayan bir $\mathbf{P}$ matrisi ile değiştirilmiştir. $\mathrm{Bu}$ $\mathbf{P}$ matrisi sonsuz basamaktaki toplam bedeli simgelemektedir. Eş. 9' a bahsedilen Lyapunov denklemi koşulu eklendiği zaman Eş. 10 elde edilmektedir. Bahsedilen süreksiz zamanlı Lyapunov denklemini çözebilmek için de Eş.11' de gösterilen ve sonsuz ufuklu LQR kontrolcüsünde tanımlanan geri besleme kazanç matrisinin kullanılması gerekmektedir. $\mathbf{P}$ matrisinin hesaplanması ve $\boldsymbol{Q}_{f}$ matrisinin yerine koyulması ile, sonlu ufuklu MPC problemi gibi, bu problem de kuadratik programlama ile çözülebilmektedir. İkili mod MPC kontrolcüsünün dikkat çeken bir detayı ise herhangi bir limitlemenin olmadığı durumlarda kontrolcünün, sonsuz ufuklu LQR kontrolcüsü ile aynı kontrol performansına sahip olmasıdır. Sisteme eklenen limitlemeler bu iki kontrolcü arasında farklılaşmalara sebep olmaktadır. 


$$
\begin{gathered}
\underbrace{\min }_{u_{0 \mid k}, \ldots, u_{N-1 \mid k}}\left\{J=\boldsymbol{x}_{N \mid k}^{T} \boldsymbol{P} \boldsymbol{x}_{N \mid k}+\sum_{j=0}^{N-1}\left(\boldsymbol{x}_{j \mid k}^{T} \boldsymbol{Q} \boldsymbol{x}_{j \mid k}+\boldsymbol{u}_{j \mid k}^{T} \boldsymbol{R} \boldsymbol{u}_{j \mid k}\right)\right\} \\
\text { Băglı olarak: } \\
\boldsymbol{x}_{k+1}=\boldsymbol{A} \boldsymbol{x}_{k}+\boldsymbol{B} \boldsymbol{u}_{k}, \\
k=0,1, \ldots, N-1 ; x_{0} \text { biliniyor } \\
\boldsymbol{Q}=\boldsymbol{Q}^{T} \geq 0, \quad \boldsymbol{Q}_{\boldsymbol{f}}=\boldsymbol{Q}_{\boldsymbol{f}}^{T} \geq 0, \quad \boldsymbol{R}=\boldsymbol{R}^{T}>0 \\
\boldsymbol{P}-\left(\boldsymbol{A}-\boldsymbol{B} \boldsymbol{K}_{\infty}\right)^{T} \boldsymbol{P}\left(\boldsymbol{A}-\boldsymbol{B} \boldsymbol{K}_{\infty}\right)=\boldsymbol{Q}+\boldsymbol{K}_{\infty}^{T} \boldsymbol{R} \boldsymbol{K}_{\infty} \\
\boldsymbol{x}_{\boldsymbol{m i n}} \leq \boldsymbol{x}_{j \mid k} \leq \boldsymbol{x}_{\boldsymbol{m a x}}, j=0,1, \ldots, N \\
\boldsymbol{u}_{\boldsymbol{m i n}} \leq \boldsymbol{u}_{j \mid k} \leq \boldsymbol{u}_{\boldsymbol{m a x}}, j=0,1, \ldots, N-1 \\
\boldsymbol{f}_{\boldsymbol{x}}^{T} \boldsymbol{x}_{j \mid k} \leq \boldsymbol{v}_{\boldsymbol{x}}, j=0,1, \ldots, N \\
\boldsymbol{f}_{\boldsymbol{u}}^{T} \boldsymbol{u}_{j \mid k} \leq \boldsymbol{v}_{\boldsymbol{u}}, j=0,1, \ldots, N-1 \\
\boldsymbol{K}_{\infty}=\left(\boldsymbol{R}+\boldsymbol{B}^{T} \boldsymbol{P}_{\infty} \boldsymbol{B}\right)^{-1} \boldsymbol{B}^{T} \boldsymbol{P}_{\infty} \boldsymbol{A}
\end{gathered}
$$

\section{Benzetim}

Çalışmasının bu kısmında, önceki kısımda tasarlanan kontrolcüler sistem dinamiği altında gözlemlenmiştir. Burada sisteme verilecek olan kontrol sinyalleri dört farklı kontrolcü ile hesaplanmış ve bu kontrol sinyalleri karşısında sistemin davranışları incelenmiştir. Burada, doğrusallaştırılmış durum uzayı ile tasarlanan kontrolcüler, doğrusal olmayan (orijinal) sistem modelinde test edilmiştir. Ek olarak gerçek hayatta ters sarkaç mekanizmasının sürekli zaman uzayında çalışıyor olması sebebiyle, benzetim yapılırken doğrusal olmayan ters sarkaç modelinin hesaplama periyodu kontrol sisteminin periyodundan çok daha küçük seçilmiştir.

Yapılan benzetim çalışması, tasarlanan dört farklı kontrolcünün davranışlarının en uygun şekilde sunulabilmesi ve tartışılabilmesi adına dört farklı parametre seçimi ile yapılmıştır. Senaryo olarak adlandırılan bu farklı parametre seçimleri kendi alt başlıkları altında incelenmiştir. Bütün senaryolarda sistemin çıktısının minimuma getirilmesi amacıyla $\boldsymbol{Q}=\boldsymbol{Q}_{\boldsymbol{f}}=\boldsymbol{C}^{T} \boldsymbol{C}$ seçimi yapılmıştır. $\mathrm{Bu}$ sayede kontrolcülerin hedefi kontrol sinyalini ve sistem çıktısını sıfıra getirmek olacaktır.

\subsection{Senaryo 1}

Yapılan benzetimde seçilen parametreler Tablo 1' de benzetim sonucu da Şekil 3' de gösterilmiştir. Yapılan benzetimde sisteme herhangi bir sinırlama tanımlanmadı ğından dolayı sonlu ufuklu LQR kontrolcü ile sonlu ufuklu MPC kontrolcü ve sonsuz ufuklu LQR kontrolcü ile ikili mod MPC kontrolcü kendi aralarında aynı kontrolü uygulamışlardır. Şekilde görüldüğü üzere sistem çıktıları olan sarkaç açısı ve araba pozisyonu, sistem girdisi olan kontrol sinyaliyle birlikte sıfıra ulaşmaktadır. Bu benzetimde dikkat çeken davranış sonlu ufuklu MPC kontrolcüsü ile sonlu ufuklu LQR kontrolcüsünün öteki iki kontrolcüye kıyasla daha düşük kontrol sinyalleri ile kontrol görevlerini tamamlamalarıdır. Kontrolcülerdeki farklı davranışın sebebi bedel fonksiyonlarındaki farklılıklardır. Sonlu ufuklu kontrolcülerde $\mathrm{N}$ değeri 50 seçildiği için kontrolcü hesaplamalarında en fazla 0,5 saniye ilerisine bakabilmektedir. $\mathrm{Bu}$ durumda sonlu ufuklu kontrolcülerde bedel fonksiyonunun minimumda olmasını engelleyen tek parametre sarkacın başlangıç açısıdır. Bu nedenle sonlu ufuklu kontrolcüler sarkacın açısını kıyasla daha hızlı sıfıra getirdi fakat araba pozisyonundaki hafif dalgalanmalara engel olamadılar. Sonsuz ufuklu kontrolcülerde hesaplanan kontrol sinyalinin daha büyük olması bedel fonksiyonlarının değerinin yüksek olmasından kaynaklanmaktadır. $\mathrm{Bu}$ kontrolcülerde $\mathrm{N}$ değerinin sonsuza gitmesinden dolayı bedel fonksiyonunda hesaba katılan durum sayısı da sonsuza gitmektedir. Bu sebeple kontrolcü daha agresif bir kontrol sinyali üretmektedir.

Ufuk seçiminin 50' den daha az olduğu durumlarda sonlu ufuklu kontrolcüler sistemi kontrol etmekte başarısız olmuşlardır. Her sistemin stabil olabilmesi için gerekli olan minimum ufuk miktarı farklıdır. Burada minimum gerekli ufuk miktarının göreceli olarak yüksek olmasının sebebi sistemin denge durumu etrafında stabil olmamasından ve eylemsizlik momentinin küçük olmasından dolayıdır. Bedel fonksiyonuna eklenen durumların sayılarının az olması, sistemi kontrol edecek doğru kontrol sinyallerinin hesaplanmasını engellemiştir. Duruma benzer olarak ufuk seçiminin daha büyük olduğu durumlarda sonlu ufuklu kontrolcülerin davranışları, sonsuz ufuklu kontrolcülerin davranışlarına daha çok benzemektedir. Bedel fonksiyonuna eklenen durumların sayısının artması, fonksiyonu sonsuz durumun hesaba katıldığı haline daha çok benzetmektedir.

Tablo 2' de gösterilen hesaplama süreleri göz önüne alındığında, hesaplaması en maliyetli olan kontrolcünün sonlu ufuklu MPC olduğu görülmektedir. Bunun sebebi bu yöntemin bahsedildiği gibi çok büyük matrislerin terslerinin alınmasını gerektirmesidir. Hesaplama süresini azaltmak amacıyla tasarlanan ikili mod MPC, ufuk sayısının daha az olması (tersinin alınması gereken matrislerinin boyutlarının küçülmesi) sebebiyle daha kısa sürede çözüm sunabilmiştir. Doğrusal yapıda olmas1 sebebiyle ve herhangi bir limiti dikkate almas gerekmemesi sebebiyle LQR kontrolcülerin çözüm sürelerinin MPC kontrolcülerin çözüm sürelerinden çok daha düşük olduğu gözlemlenmiştir. Çözüm sürelerindeki farkl1lıklara rağmen dört kontrolcü de $100 \mathrm{~Hz}$ frekanslı bir kontrol döngüsünde kullanılabilmiştir.

Tablo 1. Kontrolcü ve sistem parametreleri (Senaryo 1)

\begin{tabular}{|c|c|}
\hline $\mathrm{R}$ (kontrol sinyali ağırlık katsayısı) & 0,001 \\
\hline Kontrol sinyali limiti & Yok \\
\hline N (ufuk miktarı, hesaba katılan adım sayısı) & 50 \\
\hline İkili mod MPC N (hesaba katılan adım sayısı) & 10 \\
\hline Sarkaç açısı başlangıç değeri & $15^{\circ}$ \\
\hline Frekans (1/T) & $100 \mathrm{~Hz}$ \\
\hline
\end{tabular}

Tablo 2. Tasarlanan kontrolcülerin çözüm süreleri. Bu sonuçlar Linux Mint 20.1 işletim sistemli ve Intel $\left(\right.$ Core $^{\mathrm{TM}}$ i7-9700F CPU (a) 3.00GHz $\times 8$ islemcili bir bilgisayarda MATLAB R2020A benzetim programı aracılığ ile elde edilmiştir.

\begin{tabular}{|c|c|}
\hline Toplam benzetim süresi & 5 saniye \\
\hline Sonlu ufuklu LQR kontrolcü çözüm süresi & 0,004 saniye \\
\hline Sonsuz ufuklu LQR kontrolcü çözüm süresi & $\sim 0$ saniye \\
\hline Sonlu ufuklu MPC kontrolcü çözüm süresi & 0,536 saniye \\
\hline İkili mod MPC kontrolcü çözüm süresi & 0,348 saniye \\
\hline
\end{tabular}




\subsection{Senaryo 2}

Yapılan benzetimde seçilen parametreler Tablo 3' de benzetim sonucu da Şekil 4' de gösterilmiştir. Yapılan benzetimde tanımlanan kontrol sinyali limitlemesi sebebiyle kontrolcüler arasındaki farklılıklar ortaya çıkmaya başlamıştır. Herhangi bir limitleme olmadığı durumlarda aynı kontrol performansı gösteren ikili mod MPC ve sonsuz ufuklu LQR kontrolcüleri, ikili mod MPC kontrolcüsünün optimizasyon sırasında limitleri dikkate alması sebebiyle sonsuz ufuklu LQR kontrolcüsünden farklı bir performans göstermiştir. Sonlu ufuklu MPC kontrolcüsü ile sonlu ufuklu LQR kontrolcüsü kontrol sinyali limitlerine takılmadığından dolayı aynı kontrol performansını göstermeye devam etmişlerdir. Yapılan benzetimde, sonsuz ufuklu LQR kontrolcüsünün optimizasyonu sistem limitlerinden bağımsız olarak yapması sebebiyle yani sistem kontrol sinyalinin, hesaplamasının yapılmasından daha sonra limitlere takılması sebebiyle harekette kıyasla daha fazla dalgalanma gözlemlenmiştir. Benzer şekilde ikili mod MPC kontrolcüsünün bedel fonksiyonu içerisinde sonsuz sayıdaki basamağın bedelinin bulunması sebebiyle agresif bir kontrol sinyali üretmiş ve bu sinyal tekrar dalgalanmalara sebep olmuştur. İkili mod MPC kontrolcüsünün ufku arttırıldıkça, sonlu ufuklu MPC kontrolcüsüne daha çok benzeyen bir kontrol performansı göstermektedir fakat çözüm süresi de artmaktadır. Sonlu ufuklu LQR kontrolcüsü sadece 50 basamağın bedelini hesaplaması sebebiyle agresif bir kontrol sinyali üretmemiş ve bu kontrol sinyali sistem limitlerine takılmamıştır. Hesaplanan kontrol sinyalinin limitlere takılmamış olması sonlu ufuklu LQR kontrolcüsü ile sonlu ufuklu MPC kontrolcüsünün aynı kontrol performansı göstermesine sebep olmuştur.

Tablo 3. Kontrolcü ve sistem parametreleri (Senaryo 2).

\begin{tabular}{|c|c|}
\hline R (kontrol sinyali ağırlık katsayısı) & 0,001 \\
\hline Kontrol sinyali limiti & $7 \mathrm{~N}$ \\
\hline N (ufuk miktarı, hesaba katılan adım sayısı) & 50 \\
\hline İkili mod MPC N (hesaba katılan adım sayısı) & 20 \\
\hline Sarkaç açısı başlangıç değeri & $15^{\circ}$ \\
\hline Frekans (1/T) & $100 \mathrm{~Hz}$ \\
\hline
\end{tabular}

\subsection{Senaryo 3}

Yapılan benzetimde seçilen parametreler Tablo 4' de benzetim sonucu da Şekil 5' de gösterilmiştir. Burada kontrol sinyali limiti daha da daraltıldığında sonsuz ufuklu kontrolcülerin davranışlarında ciddi farklılıklar gözlemlenmiştir. Yapılan optimizasyonda sistem limitlerinin dikkate alınmadığı sonsuz ufuklu LQR kontrolcüsünün sistemi kontrol etmekte başarısız olduğu gözlemlenmiştir. İkili mod MPC kontrolcüsünün sistemi ancak sarkaç bir tur attıktan sonra kontrol edebildiği, sonlu ufuklu MPC kontrolcüsünün sistemi hiçbir sorun yaşamadan kontrol edebildiği gözlemlenmiştir. Sonlu ufuklu LQR kontrolcüsü limitlere takılmadığı için sonlu ufuklu MPC kontrolcüsü ile eşlenik bir kontrol karakteristiği göstermiştir. İkili mod MPC kontrolcüsünün kontrol performansı (paralel olarak çözüm süresi) N değeri arttıkça artmaktadır. İkili mod MPC kontrolcüsünün $\mathrm{N}$ değerinin 10 olduğu benzetimde, kontrolcünün sarkacı ancak iki tur attıktan sonra kontrol altına alabildiği gözlemlenmiştir.
Tablo 4. Kontrolcü ve sistem parametreleri (Senaryo 3).

\begin{tabular}{|c|c|}
\hline $\mathrm{R}$ (kontrol sinyali ağırlık katsayısı) & 0,001 \\
\hline Kontrol sinyali limiti & $5 \mathrm{~N}$ \\
\hline N (ufuk miktarı, hesaba katılan adım sayısı) & 50 \\
\hline İkili mod MPC N (hesaba katılan adım sayısı) & 20 \\
\hline Sarkaç açısı başlangıç değeri & $15^{\circ}$ \\
\hline Frekans (1/T) & $100 \mathrm{~Hz}$ \\
\hline
\end{tabular}

\subsection{Senaryo 4}

Yapılan benzetimde seçilen parametreler Tablo 5' de benzetim sonucu da Şekil 6' da gösterilmiştir. Bu senaryoda kontrol sinyali ağırlık katsayısı daha da küçültülerek kontrolcülerin daha da agresif kontrol sinyalleri üretmeleri sağlanmıştır. Kontrolcülerin daha agresif kontrol sinyalleri üretmesi bu sinyallerin limitlere daha sık takılacağı anlamına gelmektedir. Kontrol performansları gözlemlendiğinde sistem limitlerini hesaba katmasından dolayı sadece sonlu ufuklu MPC kontrolcüsünün stabil olduğu gözlemlenmiştir.

Tablo 5. Kontrolcü ve sistem parametreleri (Senaryo 4).

\begin{tabular}{|c|c|}
\hline R (kontrol sinyali ağırlık katsayısı) & 0,00001 \\
\hline Kontrol sinyali limiti & 5 \\
\hline N (ufuk miktarı, hesaba katılan adım sayısı) & 50 \\
\hline İkili mod MPC N (hesaba katılan adım sayısı) & 20 \\
\hline Sarkaç açısı başlangıç değeri & $15^{\circ}$ \\
\hline Frekans (1/T) & $100 \mathrm{~Hz}$ \\
\hline
\end{tabular}

\subsection{Farklı Sistem Frekanslarında Kontrolcü Davranışları}

Yapılan benzetimlerde tasarlanan dört kontrolcünün de seçilen ufuk sayılarında $(\mathrm{N}=50)$ ve $100 \mathrm{~Hz}$ frekansında kontrol sinyalleri üretebildikleri gösterildi. Ek olarak sonlu ufuklu kontrolcülerde belli bir sayıdan az basamakla yapılan optimizasyonlar sonucu üretilen kontrol sinyallerinin sistemi kontrol etmekte başarısız olduğu ve basamak sayısının artmasıyla birlikte çözüm süresinin de arttığı paylaşıldı. Sistemin frekansının arttırıldığı durumlarda kontrolcülerin performanslarında değişiklik gözlemlenmemiş fakat çözüm sürelerinde yüksek farklılıklar gözlemlenmiştir. Özellikle sonlu ufuklu kontrolcülerde frekansın arttığı durumlarda ufuk sayısının da arttırılmak zorunda kalınması, çözüm sürelerini çok arttırmıştır. $100 \mathrm{~Hz}$ frekansında yapılan bir kontrolde 0,5 saniye ilerisini kapsayan bir bedel fonksiyonu hesaplamak için 50 tane basamağın hesaba katılması gerekmektedir, fakat $1 \mathrm{kHz}$ frekansında yapılan bir kontrolde bu basamak sayısı 500' dür. Ufuk sayısında gerçekleşen bu artış sebebiyle MPC kontrolcüde tersi alınan matrislerin boyutları çok büyüdüğü ve MPC kontrolcülerin bu frekansta kontrol sinyali hesaplayamadığı gözlemlenmiştir. Sonlu ufuklu LQR kontrolcüsünde kullanılan Riccati denklemleri sebebiyle bir sorun yaşanmamış fakat bahsedilen MPC kontrolcülerde benzetim yapılan bilgisayarda hesaplama süresinin uzun olması sebebiyle $1 \mathrm{kHz}$ frekansında kontrol sinyali hesaplanamamıştır. 


\section{Sonuçlar}

Hemen hemen bütün kontrol uygulamalarında gözlemlendiği gibi bu çalışmada da her kontrolcünün duruma özgü olduğu ve farklı avantaj ve dezavantajları olduğu gözlemlenmiştir. Kontrol sinyallerinin fiziksel sistemdeki eyleyici limitlerini aşmadığı durumlarda sonlu ufuklu LQR kontrolcüsü ile çok işlem gücü gerekmeden, sonlu ufuklu MPC ile denk olan başarılı bir kontrol yapılabileceği gözlemlendi. Kontrol sinyalleri fiziksel sistemdeki eyleyici limitlerini aşmıyorsa ve sonlu ufuklu LQR kontrolcüsünün tasarlanması sırasında uygun bir ufuk değerinin (N) bulunmasından kaçınılmak isteniyorsa sonsuz ufuklu LQR kontrolcüsü tercih edilebilir. Unutulmamalıdır ki, sonsuz ufuklu LQR kontrolcüsü, sonlu ufuklu LQR kontrolcüsüne kıyasla daha çok dalgalanmaya sebep olmaktadır. Kontrol sinyallerinin fiziksel sistemde eyleyici limitlerine takıldığı durumlarda sonlu ufuklu MPC kontrolcüsü tercih edilebilir. MPC kontrolcüsünün sistem limitlerini göz önünde bulundurması sebebiyle bahsedilen koşullar altında LQR kontrolcülerden daha başarılı bir kontrol performansı göstermektedir. $\mathrm{Bu}$ kontrolcünün dezavantajı ise gerektirdiği işlem yüküdür. Kontrol sinyalleri fiziksel sistemde eyleyici limitlerine takılıyorsa, sonlu ufuklu MPC kontrolcüsünün tasarlanması sırasında uygun bir ufuk değerinin (N) bulunmasından kaçınılmak isteniyorsa, gerekli kontrol sinyalleri ile eyleyici limitleri arasında yüksek bir fark yoksa ve kullanılan bilgisayarın işlem gücü gerekli olan frekansta sonlu ufuklu MPC kontrolcüsü için yeterli değilse gerekli olan işlem yükünü azaltmak için ikili mod MPC kontrolcüsü tercih edilebilir.

Benzetimlenen dört senaryonun kontrolcü ve sistem parametreleri ve performans metrikleri Tablo 6' da toplu bir şekilde gösterilmiş̧tir. Her ne kadar parametre seçimi için detayl bir çalışma yapılmamış olsa da her senaryoda dört kontrolcüde de aynı parametrelerin kullanılmış olması bu kontrolcülerin kıyaslı dinamikleri hakkında çıkarımların yapılmasına olanak sağlamaktadır. Bu tablo sayesinde, ek olarak, aynı kontrolcünün farklı sistem parametreleri altında göstermiş olduğu (giriş kısıtının artması ile aşma miktarının artması gibi) davranış ve performans değişiklikleri de rahatlıkla gözlenebilmektedir.

Tablo 6. Benzetimlenen dört senaryonun kontrolcü ve sistem parametreleri ve performans metrikleri. “-” işareti girilen metrikler sarkacın tam tur atması sebebi ile anlamını yitiren metriklerdir.

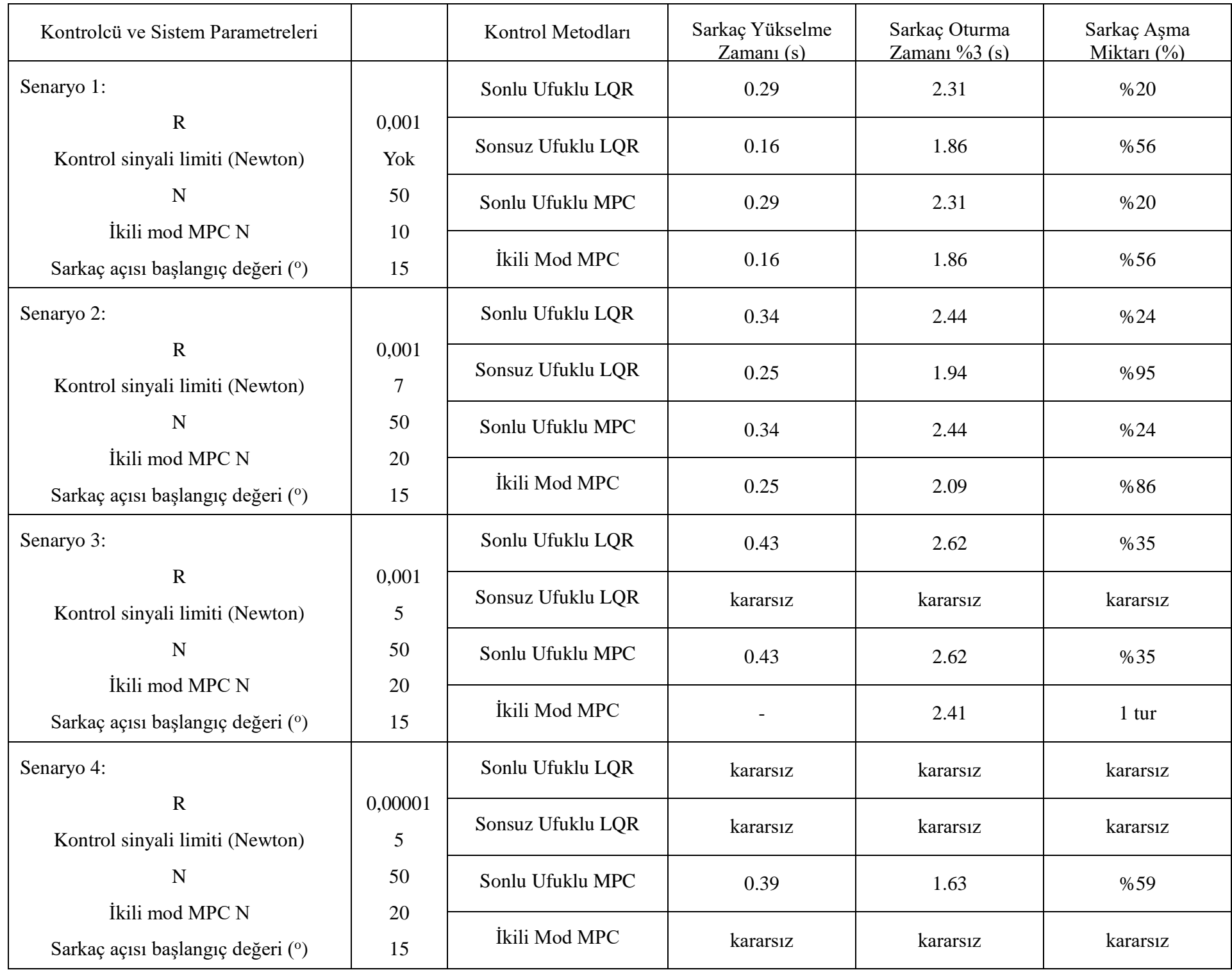




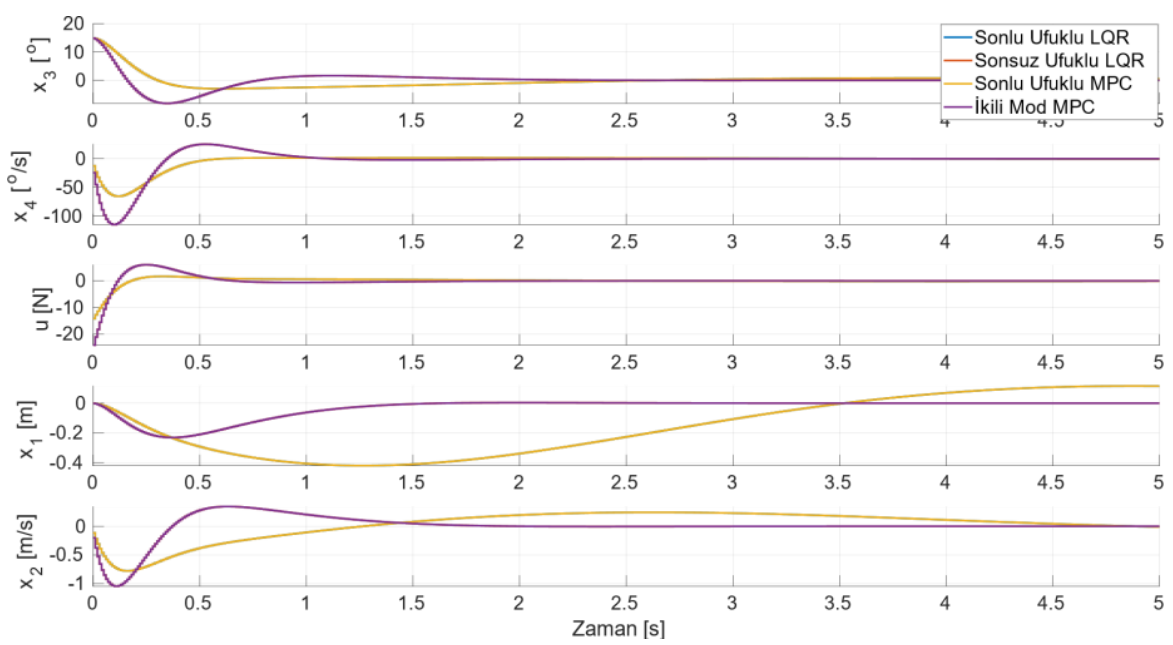

Şekil 3. Senaryo 1 olarak adlandırllan sistem ve kontrolcü durumunun benzetim sonucu.
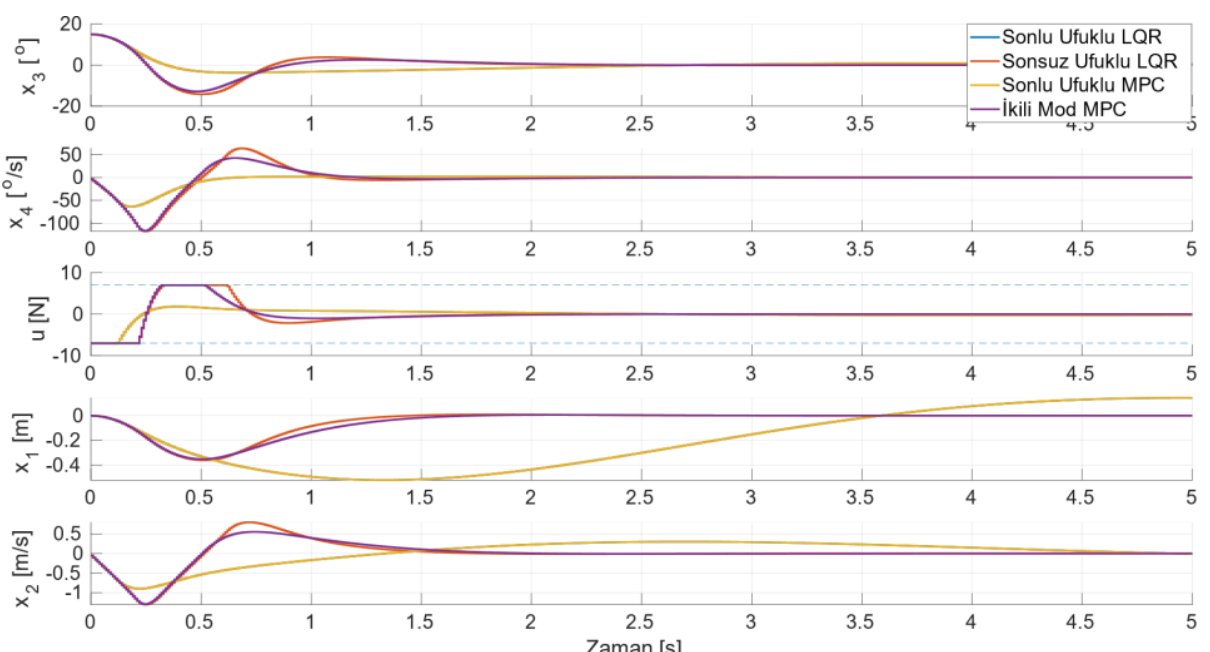

Şekil 4. Senaryo 2 olarak adlandırllan sistem ve kontrolcü durumunun benzetim sonucu.
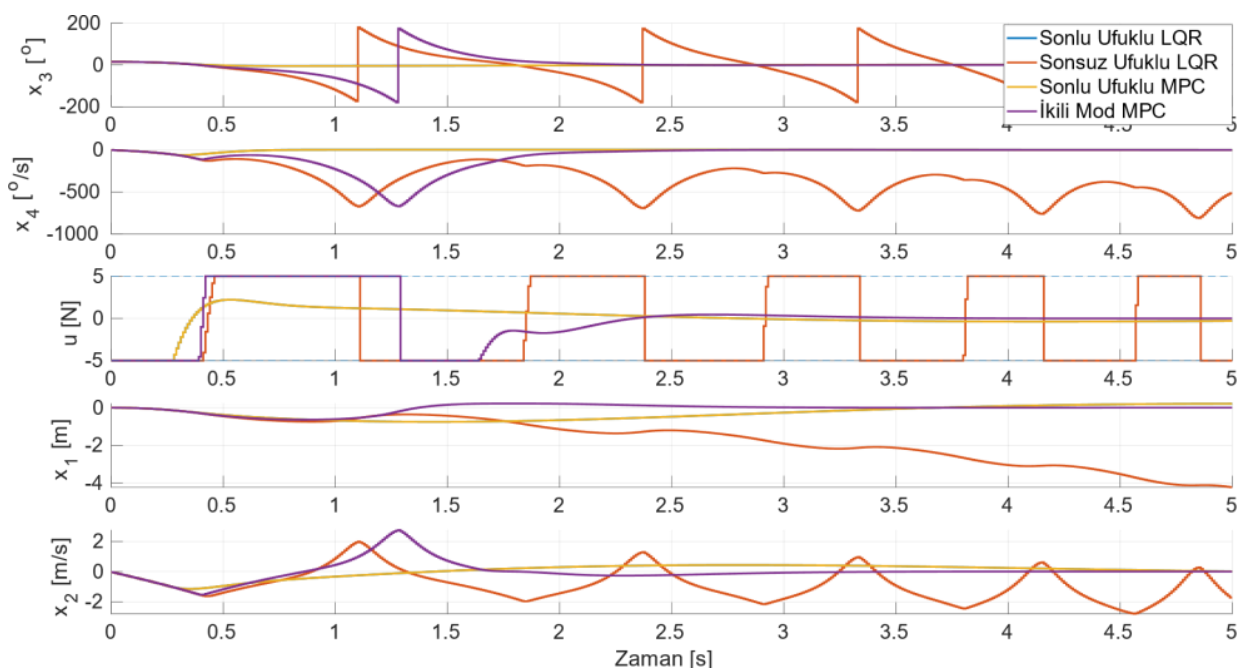

Şekil 5. Senaryo 3 olarak adlandırllan sistem ve kontrolcü durumunun benzetim sonucu. 


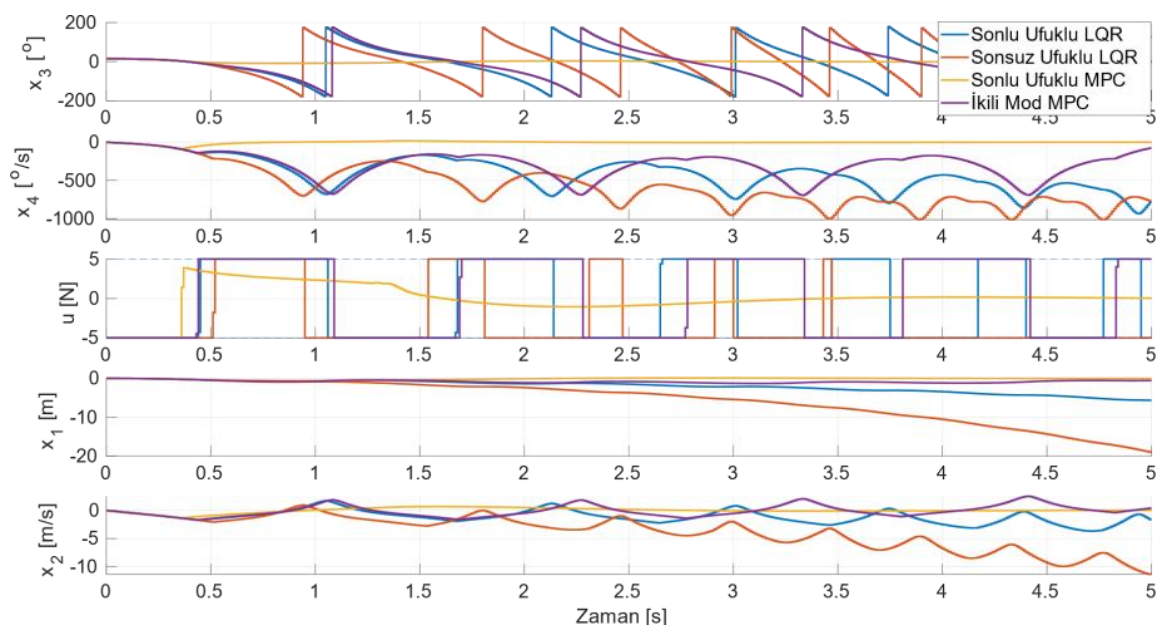

Şekil 6. Senaryo 4 olarak adlandırllan sistem ve kontrolcü durumunun benzetim sonucu.

\section{Kaynakça}

Akgul, E., Mutlu, M., Saranli, A., \& Yazicioglu, Y. (2012). A comparative evaluation of adaptive and non-adaptive Sliding Mode, LQR \& PID control for platform stabilization. 2012 IEEE International Conference on Control Applications. doi:10.1109/cca.2012.6402701

Arifianto, O., \& Farhood, M. (2015). Optimal control of a small fixed-wing UAV about concatenated trajectories. Control Engineering Practice, 40, 113-132. doi:10.1016/j.conengprac.2015.03.007

Batkovic, I., Zanon, M., Ali, M., \& Falcone, P. (2019). Real-time constrained trajectory planning and vehicle control for proactive autonomous driving with road users. 2019 18th European Control Conference (ECC). doi:10.23919/ecc.2019.8796099

Çeven, S, Albayrak, A. (2020). Çift Ters Sarkaç Sisteminin Kontrolü için PID ve LQR Kontrolcü Tasarımlarının Modellenmesi. Avrupa Bilim ve Teknoloji Dergisi, Ejosat Özel Say1 2020 (HORA), 323-330. DOI: 10.31590/ejosat.780070

Ding, Y., Pandala, A., \& Park, H. (2019). Real-time Model Predictive Control for Versatile Dynamic Motions in Quadrupedal Robots. 2019 International Conference on Robotics and Automation (ICRA). doi:10.1109/icra.2019.8793669

Frasch, J. V., Gray, A., Zanon, M., Ferreau, H. J., Sager, S., Borrelli, F., \& Diehl, M. (2013). An auto-generated nonlinear mpc algorithm for real-time obstacle avoidance of ground vehicles. 2013 European Control Conference (ECC). doi:10.23919/ecc.2013.6669836

Greer, W. B., \& Sultan, C. (2020). Shrinking horizon model predictive control method for helicopter-ship touchdown. Journal of Guidance, Control, and Dynamics, 43(5), 884900. doi:10.2514/1.g004374

Kamel, M., Burri, M., \& Siegwart, R. (2017). Linear vs Nonlinear MPC for Trajectory Tracking Applied to Rotary Wing Micro Aerial Vehicles. IFAC-PapersOnLine, 50(1), 3463-3469. doi:10.1016/j.ifacol.2017.08.849

Kirk, D. E. (2004). Optimal control theory: An introduction. Mineola, NY: Dover Publications.

Villarreal, O., Barasuol, V., Wensing, P. M., Caldwell, D. G., \& Semini, C. (2020). MPC-based controller with terrain insight for DYNAMIC legged locomotion. 2020 IEEE International Conference on Robotics and Automation (ICRA). doi:10.1109/icra40945.2020.9197312
Zhang, H., Cheng, Z., Chen, G., \& Li, C. (2015). Model predictive Flocking control for Second-order multi-agent systems with input constraints. IEEE Transactions on Circuits and Systems I: Regular Papers, 62(6), 1599-1606. doi:10.1109/tcsi.2015.2418871

Wang, J., Cui, N., \&amp; Wei, C. (2019). Optimal rocket landing guidance using convex optimization and model predictive control. Journal of Guidance, Control, and Dynamics, 42(5), 1078-1092. doi:10.2514/1.g003518 